\title{
CFD Modeling of Multi-Sized Particulate Slurry Flow through Pipe Bend
}

\author{
R. Tarodiya, S. Khullar and B. K. Gandhi ${ }^{\dagger}$ \\ Department of Mechanical and Industrial Engineering, Indian Institute of Technology, Roorkee, \\ Uttrakhand, 247667, India \\ $\dagger$ Corresponding Author : Email: bkgmefme@iitr.ac.in
}

(Received October 18, 2019; accepted December 14, 2019)

\begin{abstract}
Particle size normally varies over wide ranges in any commercial transportation of solids through the pipeline. In the present study, the three-dimensional numerical modeling of the conventional $90^{\circ}$ bend transporting multi-sized particulate slurry using granular Eulerian-Eulerian model is performed. The mixture of water and six different sizes of zinc tailing particles ranging from $37.5 \mu \mathrm{m}$ to $575 \mu \mathrm{m}$ are considered. The effect of variation in velocity and concentration on pressure drop and flow field of the multi-sized particulate slurry is investigated. The simulations are performed in the velocity range of $2.25 \mathrm{~m} / \mathrm{s}$ to $3.5 \mathrm{~m} / \mathrm{s}$ for the weighted solid concentration range of 9.82 to $44.26 \%$. The comparison of pressure drop data from the available experimental results and the present numerical modeling with multisized particulate slurry shows maximum deviation within $\pm 6 \%$. Further, the suspension behavior of different size particles in the multi-sized slurry flow inside the bend is analyzed with the variation in the flow velocity and solid concentration. The particles of different size in the multi-sized slurry showed different suspension characteristics.
\end{abstract}

Keywords: CFD; Eulerian-eulerian model; Multi-sized particle; Bend; Particle size distribution.

\section{NOMENCLATURE}

$\mathrm{C}_{\mathrm{w}} \quad$ weighted solid concentration

$\mathrm{C}_{\mathrm{v}} \quad$ solid volume fraction

$\mathrm{C}_{\mathrm{vi}} \quad$ local solid volume fraction

$\mathrm{C}_{\mathrm{vm}} \quad$ virtual mass coefficient

$\mathrm{C}_{\mathrm{L}} \quad$ lift coefficient

$\mathrm{C}_{\mathrm{d}} \quad$ drag coefficient

$d_{s} \quad s^{\text {th }}$ phase particle diameter

$\mathrm{d}_{\mathrm{q}} \quad \mathrm{q}^{\text {th }}$ phase particle diameter

D pipe internal diameter

$\mathrm{e}_{\mathrm{sq}}$ coefficient of restitution for particle collisions

g acceleration due to gravity

$\mathrm{g}_{0} \mathrm{sq}$ radial distribution function

$\mathrm{k} \quad$ turbulent kinetic energy

M momentum exchange coefficient

$\mathrm{P} \quad$ pressure

$\mathrm{Re}_{\mathrm{s}} \quad$ relative Reynolds number

$\mathrm{S} \quad$ specific gravity

$\mathrm{t}$ time

V velocity

$\mathrm{V}_{\text {sf }} \quad$ slip velocity

$\mathrm{V}_{\mathrm{r}, \mathrm{s}}$ terminal velocity for the solid

$\mathrm{V}_{\mathrm{dr}} \quad$ drift velocity

$\alpha \quad$ diffusivity $\beta \quad$ volume fraction

$\beta_{s, \max }$ maximum volumetric static settled concentration taken as 0.35

$\rho$ density

$\lambda_{s} \quad$ bulk viscosity of solids

$\Theta_{s} \quad$ granular temperature of solid phase

$\mu \quad$ shear viscosity

$\mu_{t} \quad$ turbulent viscosity

$\varepsilon \quad$ turbulence dissipation rate

$I_{2 D}$ second invariant of the deviatoric stress tensor for solid phase

$\sigma_{s f} \quad$ dispersion Prandtl number taken as 0.75

$\Phi \quad$ internal friction angle taken as $30^{\circ}$

$\bar{I} \quad$ identity tensor

$\tau \quad$ viscous stress tensor

$\overline{\tau_{t}} \quad$ Reynolds stress tensor

\begin{tabular}{ll}
\multicolumn{2}{l}{ Subscripts } \\
$\mathrm{f}$ & fluid \\
$\mathrm{s}$ & solid \\
s,col & collisional part \\
s,kin & kinetic part \\
s,fr & frictional part
\end{tabular}




\section{INTRODUCTION}

Hydraulic transportation of solids is generally adopted in many process industries such as mineral, chemical, and power (Tarodiya and Gandhi, 2019). Two important aspects are generally considered while designing the slurry pipelines: the amount of head loss due to the presence of suspended solids and the service life due to erosive wear.

To provide the flexibility in the routing of the pipeline, geometries like bends and tee sections are used. The flow field inside the bend is very complex due to its curved flow path. Secondary flows are developed which strongly affect the flow characteristics of the particles inside the bend (Kaushal et al. 2012; Nayak et al. 2017). Additionally, during slurry transportation, the pressure drop and flow characteristics are influenced by: (i) the particle properties like size, shape, and distribution, (ii) carrier fluid properties like viscosity and flow velocity, and (iii) slurry properties like the amount of solid loading and its rheological behavior. The particle size is one of the dominating parameters affecting the pressure drop and particle flow characteristics (Mukhtar et al. 1995) across the bend. Many investigators (Mukhtar et al. 1995; Mishra et al. 1998a; Kaushal and Tomita, 2002; Verma et al. 2006; Singh et al. 2019) experimentally determined the pressure drop and flow characteristics of the solids inside the bend. Numerical approach has also been used to understand the complexity of flow over a wide range of parameters which are difficult to obtain through the experiments. Kaushal et al. (2012) numerically investigated the pressure drop in a horizontal pipeline transporting high concentrated solid-liquid mixture using granular Mixture and granular Eulerian-Eulerian multiphase models of the CFD code Fluent. The simulations were performed using equisized particles of glass bead size of $125 \mu \mathrm{m}$ in water. They reported better accuracy for EulerianEulerian model to predict the pressure drop and concentration distribution with respect to the experimental data. Further, Kaushal et al. (2013) used granular Eulerian-Eulerian model to simulate the pressure drop and concentration distribution in $90^{\circ}$ horizontal bend transporting slurry of equisized sand particles of size $450 \mu \mathrm{m}$ in CFD code Fluent. They reported that the suspension of the particles is good at the downstream of the bend and improves further with increase in downstream distance from the bend exit. Messa and Malavasi (2014) performed the numerical simulation to predict the particle distribution in $90^{\circ}$ horizontal bend with equisized particles. They discussed that the presence of secondary flows significantly affects the distribution of solids. The concentration distribution is nonhomogeneous at different cross-sections inside the bend. Nayak et al. (2017) investigated the transport characteristics for the slurry in an $180^{\circ}$ return bend. They performed the simulations in Fluent with water and equisized fly ash particles of size $13 \mu \mathrm{m}$. They also observed the variation in the distribution of solids across the bend due to secondary flows. Singh et al. (2018) numerically investigated the effect of variation in head loss due to solid-liquid flow with the change in the radius ratio of the $90^{\circ}$ pipe bend. They performed the simulations using CFD code Fluent with water and equisized fly ash particles of size $16 \mu \mathrm{m}$. Literature review reveals that the efforts were made to numerically investigate the pressure drop and flow characteristics of solids inside the bend under different operating conditions. However, these studies were restricted to the slurry of equisized particles. In commercial slurry transportation, generally, the particle size is varied up to three orders of magnitude (Kaushal and Tomita, 2002; Verma et al. 2006). Variation in particle kinetics due to the wide variation in particle size distribution (PSD) may significantly affect the head loss and particle flow characteristics which may not be captured with the equi-size particles. Numerical simulations with multisized particulate slurry may provide a better picture of actual slurry flow inside the bend which may help the designers in designing the slurry pipeline for better flow stability.

The aim of the present study is to establish a numerical model for the simulation of multi-sized particulate slurry flows. For this, the threedimensional modeling of a $90^{\circ}$ horizontal pipe bend of diameter $105 \mathrm{~mm}$ handling multi-sized particulate slurry is performed. The simulations are performed using the granular Eulerian-Eulerian multiphase model of Fluent 15.0. Six different sizes of zinc tailings particles $\left(\mathrm{S}_{\mathrm{s}}=2.85\right)$ of mean diameter ranging from $37.5 \mu \mathrm{m}$ to $575 \mu \mathrm{m}$ are used with water as the carrier fluid for the simulation. The pressure drop is estimated in the velocity range of 2.25-3.5 m/s and overall weighted solid concentration is varied from 9.82 to $44.26 \%$. The numerical model is validated with the experimental data of Mishra et al. (1998a). Further, the flow field of the multisized particles is analyzed across the bend at different locations with the variation in flow velocity and efflux concentration.

\section{Mathematical Model}

In the present study, granular Eulerian-Eulerian model is used for modeling the multi-sized particulate slurry flow inside the pipe bend. It allows for modeling of unlimited number of secondary phases. However, the computational cost increases with the increase in number of secondary phases, as separate governing equations are required to solve for each phase. In this the solid (s) and fluid (f) phases, are considered to be interpenetrating continua, so that $\beta_{\mathrm{s}}+\beta_{\mathrm{f}}=1$, where $\beta_{\mathrm{s}}$ and $\beta_{\mathrm{f}}$ are the volumetric concentrations of solid and fluid phases, respectively. The conservation of mass and momentum is satisfied for each phase individually. The coupling between them is achieved with the interphase exchange coefficients.

\subsection{Continuity Equation}

$\nabla \cdot\left(\beta_{\mathrm{n}} \rho_{\mathrm{n}} \overrightarrow{\mathrm{V}}_{\mathrm{n}}\right)=0$, where, $\mathrm{n}$ is either s or $\mathrm{f}$. 


\subsection{Momentum Equation}

For fluid phase:

$$
\begin{aligned}
& \nabla \cdot\left(\beta_{\mathrm{f}} \rho_{\mathrm{f}} \overrightarrow{\mathrm{V}}_{\mathrm{f}} \overrightarrow{\mathrm{V}}_{\mathrm{f}}\right)=-\beta_{\mathrm{f}} \nabla \mathrm{P}+\beta_{\mathrm{f}} \rho_{\mathrm{f}} \overrightarrow{\mathrm{g}}+ \\
& \nabla \cdot\left(\bar{\tau}_{\mathrm{f}}+\bar{\tau}_{\mathrm{t}, \mathrm{f}}\right)+\mathrm{M}_{\mathrm{sf}}\left(\overrightarrow{\mathrm{V}}_{\mathrm{s}}-\overrightarrow{\mathrm{V}}_{\mathrm{f}}\right)+ \\
& \mathrm{C}_{\mathrm{vm}} \beta_{\mathrm{s}} \rho_{\mathrm{f}}\left(\overrightarrow{\mathrm{V}}_{\mathrm{s}} . \nabla \overrightarrow{\mathrm{V}}_{\mathrm{s}}-\overrightarrow{\mathrm{V}}_{\mathrm{f}} \cdot \nabla \overrightarrow{\mathrm{V}}_{\mathrm{f}}\right)+ \\
& \mathrm{C}_{\mathrm{L}} \beta_{\mathrm{s}} \rho_{\mathrm{f}}\left(\overrightarrow{\mathrm{V}}_{\mathrm{f}}-\overrightarrow{\mathrm{V}}_{\mathrm{s}}\right) \times\left(\nabla \times \overrightarrow{\mathrm{V}}_{\mathrm{f}}\right)
\end{aligned}
$$

For solid phase:

$$
\begin{aligned}
& \nabla \cdot\left(\beta_{\mathrm{s}} \rho_{\mathrm{s}} \overrightarrow{\mathrm{V}}_{\mathrm{s}} \overrightarrow{\mathrm{V}}_{\mathrm{s}}\right)=-\beta_{\mathrm{s}} \nabla \mathrm{P}+\beta_{\mathrm{s}} \rho_{\mathrm{s}} \overrightarrow{\mathrm{g}}-\nabla \mathrm{P}_{\mathrm{s}}+ \\
& \nabla \cdot\left(\tau_{\mathrm{s}}+\tau_{\mathrm{t}, f}\right)+\mathrm{M}_{\mathrm{fs}}\left(\overrightarrow{\mathrm{V}}_{\mathrm{f}}-\overrightarrow{\mathrm{V}}_{\mathrm{s}}\right)+ \\
& \mathrm{M}_{\mathrm{sq}}\left(\overrightarrow{\mathrm{V}}_{\mathrm{s}}-\overrightarrow{\mathrm{V}}_{\mathrm{q}}\right)+ \\
& \mathrm{C}_{\mathrm{vm}} \beta_{\mathrm{s}} \rho_{\mathrm{f}}\left(\overrightarrow{\mathrm{V}}_{\mathrm{f}} \cdot \nabla \overrightarrow{\mathrm{V}}_{\mathrm{f}}-\overrightarrow{\mathrm{V}}_{\mathrm{s}} \cdot \nabla \overrightarrow{\mathrm{V}}_{\mathrm{s}}\right)+ \\
& \mathrm{C}_{\mathrm{L}} \beta_{\mathrm{s}} \rho_{\mathrm{f}}\left(\overrightarrow{\mathrm{V}}_{\mathrm{s}}-\overrightarrow{\mathrm{V}}_{\mathrm{f}}\right) \times\left(\nabla \times \overrightarrow{\mathrm{V}}_{\mathrm{f}}\right)
\end{aligned}
$$

Where, $\nabla \mathrm{P}$ is the static pressure gradient, $\rho \vec{g}$ is the body force, $\nabla \mathrm{P}_{\mathrm{s}}$ is the solid pressure gradient, $\mathrm{C}_{\mathrm{vm}} \beta_{\mathrm{s}} \rho_{\mathrm{f}}\left(\overrightarrow{\mathrm{V}}_{\mathrm{f}} \cdot \nabla \overrightarrow{\mathrm{V}}_{\mathrm{f}}-\overrightarrow{\mathrm{V}}_{\mathrm{s}} \cdot \nabla \overrightarrow{\mathrm{V}}_{\mathrm{s}}\right)$ is the virtual mass force, and $C_{L} \beta_{s} \rho_{f}\left(\vec{V}_{f}-\vec{V}_{s}\right) \times\left(\nabla \times \vec{V}_{f}\right)$ is lift force acting on the particles. The coefficient of virtual mass force $\left(\mathrm{C}_{\mathrm{vm}}\right)$ and lift coefficient $\left(\mathrm{C}_{\mathrm{L}}\right)$ are taken as 0.5 (Gopaliya and Kaushal, 2015).

For each solid phase, the solid pressure $\left(\mathrm{P}_{\mathrm{s}}\right)$ is estimated from the correlation given by Lun et al. (1984), and expressed as:

$$
\begin{aligned}
& \mathrm{P}_{\mathrm{s}}=\beta_{\mathrm{s}} \rho_{\mathrm{s}} \Theta_{\mathrm{s}}+ \\
& \sum_{\mathrm{q}=1}^{\mathrm{N}} 2 \frac{\mathrm{d}_{\mathrm{sq}}^{3}}{\mathrm{~d}_{\mathrm{s}}^{3}}\left(1+\mathrm{e}_{\mathrm{sq}}\right) \rho_{\mathrm{s}} \beta_{\mathrm{s}} \beta_{\mathrm{q}} \mathrm{g}_{0, \mathrm{sq}} \Theta_{\mathrm{s}}
\end{aligned}
$$

Where $\mathrm{d}_{\mathrm{sq}}\left(=\mathrm{d}_{\mathrm{s}} / 2+\mathrm{d}_{\mathrm{q}} / 2\right)$ is the average of the $\mathrm{s}^{\text {th }}$ and the $\mathrm{q}^{\text {th }}$ phase particle diameters $\mathrm{d}_{\mathrm{s}}$ and $\mathrm{d}_{\mathrm{q}}, \mathrm{g}_{0, \mathrm{sq}}$ is the radial distribution function for solid phases and calculated as (Lun et al. 1984):

$$
\mathrm{g}_{0, \mathrm{sq}}=\left[1-\left(\frac{\beta_{\mathrm{s}}}{\beta_{\mathrm{s}, \max }}\right)^{\frac{1}{3}}\right]^{-1}+\frac{1}{2} \mathrm{~d}_{\mathrm{s}} \sum_{\mathrm{q}=1}^{\mathrm{N}+1} \frac{\beta_{\mathrm{q}}}{\mathrm{d}_{\mathrm{q}}}
$$

The granular temperature $\left(\Theta_{\mathrm{s}}\right)$ for solid phase is calculated using the equation (Ding and Gidaspow, 1990) as,

$\frac{3}{2}\left[\frac{\partial}{\partial \mathrm{t}}\left(\rho_{\mathrm{s}} \beta_{\mathrm{s}} \Theta_{\mathrm{s}}\right)+\nabla \cdot\left(\rho_{\mathrm{s}} \beta_{\mathrm{s}} \overrightarrow{\mathrm{V}}_{\mathrm{s}} \Theta_{\mathrm{s}}\right)\right]=$

$\left(-\mathrm{p}_{\mathrm{s}}^{\overline{\mathrm{I}}+\bar{\tau}_{\mathrm{s}}}\right): \nabla \overrightarrow{\mathrm{V}}_{\mathrm{s}}+\nabla \cdot\left(\mathrm{k}_{\Theta_{\mathrm{s}}} \nabla \Theta_{\mathrm{s}}\right)-\gamma_{\Theta_{\mathrm{s}}}+\phi_{\mathrm{fs}}$

Where, $\left(-\mathrm{p}_{\mathrm{s}} \overline{\overline{\mathrm{I}}}+\tau_{\mathrm{s}}\right): \nabla \overrightarrow{\mathrm{V}}_{\mathrm{s}}$ is the creation of fluctuation energy due to shear in the solid phase, $\mathrm{k}_{\Theta_{\mathrm{s}}} \nabla \Theta_{\mathrm{s}}$ is the diffusion flux of granular energy.

The term $k_{\Theta_{\mathrm{s}}}$ is the diffusion coefficient given by Syamlal et al. (1993),

$$
\mathrm{k}_{\Theta_{\mathrm{s}}}=\frac{15 \mathrm{~d}_{\mathrm{s}} \rho_{\mathrm{s}} \beta_{\mathrm{s}} \sqrt{\Theta_{\mathrm{s}} \pi}}{4(41-33 \eta)}\left[\begin{array}{l}
1+ \\
\frac{12}{5} \eta^{2}(4 \eta-3) \beta_{\mathrm{s}} \mathrm{g}_{0, \mathrm{sq}}+ \\
\frac{16}{15 \pi}(41-33 \eta) \eta \beta_{\mathrm{s}} \mathrm{g}_{0, \mathrm{sq}}
\end{array}\right]
$$

Where, $\eta=\frac{1}{2}\left(1+\mathrm{e}_{\mathrm{sq}}\right)$

The rate of energy dissipation within the solid phase due to a collision between particles $\left(\gamma_{\Theta s}\right)$ is calculated using the correlation (Lun et al. 1984) as,

$\gamma_{\Theta_{\mathrm{s}}}=\frac{12\left(1-\mathrm{e}_{\mathrm{sq}}^{2}\right) \mathrm{g}_{0, \mathrm{sq}}}{\mathrm{d}_{\mathrm{s}} \sqrt{\pi}} \rho_{\mathrm{s}} \beta_{\mathrm{s}}^{2} \Theta_{\mathrm{s}}^{3 / 2}$

The transfer of kinetic energy of random fluctuation in particle velocity from the solid phase to the fluid phase $\left(\phi_{\mathrm{fs}}\right)$ is calculated using the correlation (Fluent, 2006) given as,

$\phi_{\mathrm{fs}}=-3 \mathrm{M}_{\mathrm{fs}} \Theta_{\mathrm{s}}$

In Eqs. (2) and (3), $\tau_{\mathrm{s}}$ and $\tau_{\mathrm{f}}$ are the stress-strain tensors for solid and fluid phase respectively, and are expressed as

$$
\begin{aligned}
& = \\
& \tau_{\mathrm{s}}=\beta_{\mathrm{s}} \mu_{\mathrm{s}}\left(\nabla \overrightarrow{\mathrm{V}}_{\mathrm{s}}+\nabla \overrightarrow{\mathrm{V}}_{\mathrm{s}}^{\mathrm{T}}\right)+ \\
& \beta_{\mathrm{s}}\left(\lambda_{\mathrm{s}}-\frac{2}{3} \mu_{\mathrm{s}}\right) \nabla \cdot \overrightarrow{\mathrm{V}}_{\mathrm{s}} \mathrm{I}
\end{aligned}
$$

and

$$
\bar{\tau}_{\mathrm{f}}=\beta_{\mathrm{f}} \mu_{\mathrm{f}}\left(\nabla \overrightarrow{\mathrm{V}}_{\mathrm{f}}+\nabla \overrightarrow{\mathrm{V}}_{\mathrm{f}}^{\mathrm{T}}\right)
$$

Where $\lambda_{\mathrm{s}}$ is the bulk viscosity of the solid particles calculated using the correlation (Lun et al. 1984):

$\lambda_{\mathrm{s}}=\frac{4}{3} \beta_{\mathrm{s}} \rho_{\mathrm{s}} \mathrm{d}_{\mathrm{s}} \mathrm{g}_{0, \mathrm{sq}}\left(1+\mathrm{e}_{\mathrm{sq}}\right)\left(\frac{\Theta_{\mathrm{s}}}{\pi}\right)^{1 / 2}$

and $\mu_{\mathrm{s}}$ is the shear viscosity of solids defined as (Fluent, 2006)

$$
\mu_{\mathrm{s}}=\mu_{\mathrm{s}, \mathrm{col}}+\mu_{\mathrm{s}, \mathrm{kin}}+\mu_{\mathrm{s}, \mathrm{fr}}
$$

The collisional, kinetic and frictional part of the shear viscosity of solid phase is calculated from the correlations of the form (Syamlal et al. 1993; Gidaspow et al. 1992; Schaeffer, 1987):

$$
\mu_{\mathrm{s}, \mathrm{col}}=\frac{4}{5} \beta_{\mathrm{s}} \rho_{\mathrm{s}} \mathrm{d}_{\mathrm{s}} \mathrm{g}_{0, \mathrm{sq}}\left(1+\mathrm{e}_{\mathrm{sq}}\right)\left(\frac{\Theta_{\mathrm{s}}}{\pi}\right)^{1 / 2}
$$

$\mu_{\mathrm{s}, \text { kin }}=\frac{\beta_{\mathrm{s}} \rho_{\mathrm{s}} \mathrm{d}_{\mathrm{s}} \sqrt{\Theta_{\mathrm{s}} \pi}}{6\left(3-\mathrm{e}_{\mathrm{sq}}\right)} \mathrm{x}$

$\left[1+\frac{2}{5}\left(1+\mathrm{e}_{\mathrm{sq}}\right)\left(3 \mathrm{e}_{\mathrm{sq}}-1\right) \beta_{\mathrm{s}} \mathrm{g}_{0, \mathrm{sq}}\right]$ 
$\mu_{\mathrm{s}, \mathrm{fr}}=\frac{\mathrm{P}_{\mathrm{s}} \sin \phi}{2 \sqrt{\mathrm{I}_{2 \mathrm{D}}}}$

The Reynolds stress tensor for a fluid phase in the Eqs. (2) and (3) is estimated as (Fluent, 2006):

$$
\begin{aligned}
& \bar{\tau}_{\mathrm{t}, \mathrm{f}}=-\frac{2}{3}\left(\rho_{\mathrm{f}} \mathrm{k}_{\mathrm{f}}+\rho_{\mathrm{f}} \mu_{\mathrm{t}, \mathrm{f}} \nabla \overrightarrow{\mathrm{V}}_{\mathrm{f}}\right) \overline{\mathrm{I}}+ \\
& \rho_{\mathrm{f}} \mu_{\mathrm{t}, \mathrm{f}}\left(\nabla \overrightarrow{\mathrm{V}}_{\mathrm{f}}+\nabla \overrightarrow{\mathrm{V}}_{\mathrm{f}}^{\mathrm{T}}\right)
\end{aligned}
$$

where $\mu_{\mathrm{t}, \mathrm{f}}$ is the turbulent viscosity, computed from

$\mu_{\mathrm{t}, \mathrm{f}}=\rho_{\mathrm{f}} \mathrm{C}_{\mu} \frac{\mathrm{k}_{\mathrm{f}}{ }^{2}}{\varepsilon_{\mathrm{f}}} \quad$ where, $\mathrm{C}_{\mu}=0.09$

The prediction of turbulent kinetic energy $\mathrm{kf}_{\mathrm{f}}$ and its rate of dissipation $\varepsilon$ for the fluid phase are obtained from the following transport equation

$$
\begin{aligned}
& \nabla .\left(\beta_{\mathrm{f}} \rho_{\mathrm{f}} \overrightarrow{\mathrm{V}}_{\mathrm{f}} \mathrm{k}_{\mathrm{f}}\right)=\nabla \cdot\left(\beta_{\mathrm{f}} \frac{\mu_{\mathrm{t}, \mathrm{f}}}{\sigma_{\mathrm{k}}} \nabla \mathrm{k}_{\mathrm{f}}\right)+ \\
& \beta_{\mathrm{f}} \mathrm{G}_{\mathrm{k}, \mathrm{f}}-\beta_{\mathrm{f}} \rho_{\mathrm{f}} \varepsilon_{\mathrm{f}}+\beta_{\mathrm{f}} \rho_{\mathrm{f}} \prod_{\mathrm{kf}} \\
& \nabla \cdot\left(\beta_{\mathrm{f}} \rho_{\mathrm{f}} \overrightarrow{\mathrm{V}}_{\mathrm{f}} \varepsilon_{\mathrm{f}}\right)=\nabla \cdot\left(\beta_{\mathrm{f}} \frac{\mu_{\mathrm{t}, \mathrm{f}}}{\sigma_{\varepsilon}} \nabla \varepsilon_{\mathrm{f}}\right)+ \\
& \beta_{\mathrm{f}} \frac{\varepsilon_{\mathrm{f}}}{\mathrm{k}_{\mathrm{f}}}\left(\mathrm{C}_{1 \varepsilon} \mathrm{G}_{\mathrm{k}, \mathrm{f}}-\mathrm{C}_{2 \varepsilon} \rho_{\mathrm{f}} \varepsilon_{\mathrm{f}}\right)+\beta_{\mathrm{f}} \rho_{\mathrm{f}} \prod_{\varepsilon f}
\end{aligned}
$$

where, $\mathrm{G}_{\mathrm{k}, \mathrm{f}}$ is the production of turbulent kinetic energy in the flow estimated as

$\mathrm{G}_{\mathrm{k}, \mathrm{f}}=\mu_{\mathrm{t}, \mathrm{f}}\left(\nabla \overrightarrow{\mathrm{V}}_{\mathrm{f}}+\nabla \overrightarrow{\mathrm{V}}_{\mathrm{f}}^{\mathrm{T}}\right): \nabla \overrightarrow{\mathrm{V}}_{\mathrm{f}}$

$\prod_{\mathrm{kf}}$ and $\prod_{\varepsilon \mathrm{f}}$ represent the influence of the solid phase on the fluid phase computed from

$$
\begin{aligned}
& \prod_{\mathrm{kf}}=\frac{\mathrm{K}_{\mathrm{fs}}}{\beta_{\mathrm{f}} \rho_{\mathrm{f}}}\left(\mathrm{k}_{\mathrm{sf}}-2 \mathrm{k}_{\mathrm{f}}+\overrightarrow{\mathrm{V}}_{\mathrm{sf}} \cdot \overrightarrow{\mathrm{V}}_{\mathrm{dr}}\right) \\
& \prod_{\varepsilon \mathrm{f}}=\mathrm{C}_{3 \varepsilon} \frac{\varepsilon_{\mathrm{f}}}{\mathrm{k}_{\mathrm{f}}} \prod_{\mathrm{kf}}
\end{aligned}
$$

Where, $\mathrm{C}_{1 \varepsilon}=1.44, \mathrm{C}_{2 \varepsilon}=1.92, \mathrm{C}_{3 \varepsilon}=1.2, \sigma_{\mathrm{k}}=1.0$, $\sigma_{\varepsilon}=1.3$

$\vec{V}_{\text {sf is }}$ the slip velocity, the relative velocity between the fluid phase and solid phase given by

$$
\overrightarrow{\mathrm{V}}_{\mathrm{sf}}=\overrightarrow{\mathrm{V}}_{\mathrm{s}}-\overrightarrow{\mathrm{V}}_{\mathrm{f}}
$$

$\overrightarrow{\mathrm{V}}_{\mathrm{dr}}$ is the drift velocity given by

$$
\overrightarrow{\mathrm{V}}_{\mathrm{dr}}=-\left(\frac{\alpha_{\mathrm{s}}}{\sigma_{\mathrm{sf}} \beta_{\mathrm{s}}} \nabla \beta_{\mathrm{s}}-\frac{\alpha_{\mathrm{f}}}{\sigma_{\mathrm{sf}} \beta_{\mathrm{f}}} \nabla \beta_{\mathrm{f}}\right)
$$

The momentum exchange coefficient between the fluid and solid phase in the Eqs. (2) and (3) is estimated as (Fluent, 2006):
$M_{s f}=M_{f s}=\frac{3 \beta_{s} \beta_{f} \rho_{f}}{4 V_{r, s}^{2} d_{s}} C_{D}\left(\frac{R_{s}}{V_{r, s}}\right)\left|\vec{V}_{s}-\vec{V}_{f}\right|$

Where, $\mathrm{C}_{\mathrm{D}}$ is the drag coefficient given by Dalla Valle (1948):

$\mathrm{C}_{\mathrm{D}}=\left(0.63+\frac{4.8}{\sqrt{\mathrm{Re}_{\mathrm{s}} / \mathrm{V}_{\mathrm{r}, \mathrm{s}}}}\right)^{2}$

$\mathrm{Re}_{\mathrm{s}}$ is the relative Reynolds number between solid and liquid phase is given by:

$\operatorname{Re}_{\mathrm{s}}=\frac{\rho_{\mathrm{f}} \mathrm{d}_{\mathrm{s}}\left|\overrightarrow{\mathrm{V}}_{\mathrm{s}}-\overrightarrow{\mathrm{V}}_{\mathrm{f}}\right|}{\mu_{\mathrm{f}}}$

$\mathrm{V}_{\mathrm{r}, \mathrm{s}}$ is the terminal velocity correlation for the solid phase is given by (Garside and Al-Dibouni, 1977):

$\mathrm{V}_{\mathrm{r}, \mathrm{s}}=0.5\left(\sqrt{\frac{\mathrm{A}-0.06 \mathrm{Re}_{\mathrm{s}}+}{\left(0.06 \mathrm{Re}_{\mathrm{s}}\right)^{2}+}}\right)$

where,

$$
\begin{array}{ll}
\mathrm{A}=\beta_{\mathrm{f}}^{4.14} ; \mathrm{B}=0.8 \beta_{\mathrm{f}}^{1.28} & \text { for } \beta_{\mathrm{f}} \leq 0.85 \\
\mathrm{~A}=\beta_{\mathrm{f}}^{4.14} ; \mathrm{B}=\beta_{\mathrm{f}}^{2.65} & \text { for } \beta_{\mathrm{f}}>0.85
\end{array}
$$

The momentum exchange coefficient between the solid phases in the Eq. (3) is estimated as (Syamlal, 1987):

$$
\begin{aligned}
& \mathrm{M}_{\mathrm{sq}}=3\left(1+\mathrm{e}_{\mathrm{sq}}\right)\left(\frac{\pi}{2}+\mathrm{C}_{\mathrm{fr}, \mathrm{sq}} \frac{\pi^{2}}{8}\right) \mathrm{x} \\
& \frac{\beta_{\mathrm{s}} \rho_{\mathrm{s}} \beta_{\mathrm{q}} \rho_{\mathrm{q}}\left(\mathrm{d}_{\mathrm{s}}+\mathrm{d}_{\mathrm{q}}\right)^{2} \mathrm{~g}_{0, \mathrm{sq}}}{2 \pi\left(\rho_{\mathrm{s}} \mathrm{d}_{\mathrm{s}}^{3}+\rho_{\mathrm{q}} \mathrm{d}_{\mathrm{q}}^{3}\right)}\left|\overrightarrow{\mathrm{V}}_{\mathrm{s}}-\overrightarrow{\mathrm{V}}_{\mathrm{q}}\right|
\end{aligned}
$$

\section{Numerical Procedure}

\subsection{Geometry and Meshing}

The simulations are performed on a pipe with 105 $\mathrm{mm}$ internal diameter (D), 90 degree conventional bend with an area ratio of 1 which is equal to the radius ratio of $4(\mathrm{R} / \mathrm{r}=4)$, similar to the bend geometry used by Mishra et al. (1998a). In order to better represent the flow inside the bend, a $2.1 \mathrm{~m}$ (20D) horizontal pipe upstream to the bend and 1.26 $\mathrm{m}$ (12D) horizontal pipe downstream to the bend are used. The bend is placed horizontally in $(\mathrm{x}, \mathrm{z})$ plane with the origin at the pipe inlet $(0,0,0)$. The direction of flow at the inlet is in the positive $\mathrm{x}$ direction. The direction of flow at the outlet is in the positive z-direction. ANSYS ICEM is used to generate O-type structured mesh of hexahedral elements. A cross-sectional view of the mesh at the inlet and the enlarged view of pipe bend are presented in Fig. 1(a) and Fig. 1(b), respectively. Mesh independency check is also performed to keep the balance between the computational time and the 
accuracy of the simulation. For this purpose, five different size mesh geometries named as M1 to M5 are generated with mesh elements in the range of 0.96 million to 9.8 million. The simulation with different meshes is performed for the flowing fluid water at a velocity of $2.25 \mathrm{~m} / \mathrm{s}$. The predicted pressure drop between 2D upstream and 2D downstream of the bend inlet and outlet is used for validation with the experimental data of Mishra et al. (1998a). Figure 2 shows the predicted pressure drop from different meshes and the deviation (in brackets) from the experimental data. It is observed that the pressure drop decreases with the mesh M1 to M5. The predicted value from mesh M3 shows less than $1 \%$ variation as compared to the mesh M5. Therefore, M3 mesh of 2.2 million hexahedra elements with minimum orthogonal quality of 0.75 is selected for the present study. The obtained $y+$ value of the numerical model is in the range of 20 to 50. The standard wall function approach is used for modeling the flow near the wall region. It helps in more precise calculation of near-wall shear stresses for both liquid and solid phases (Kaushal et al. 2012).

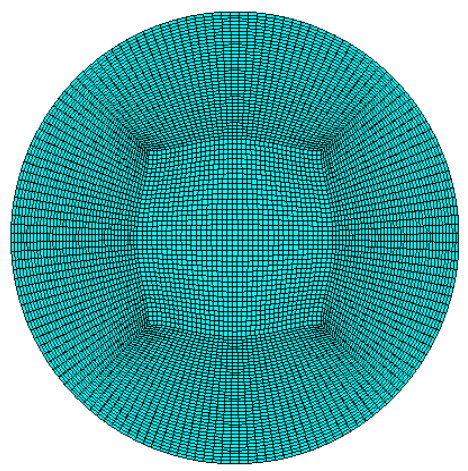

(a) Cross-sectional view at pipe inlet

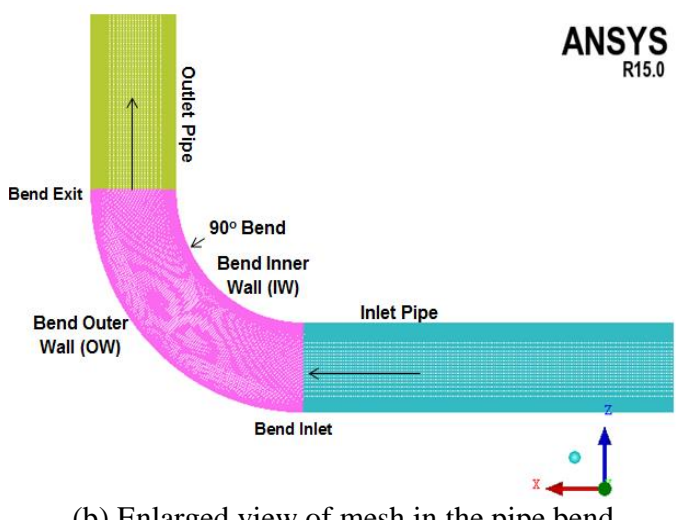

(b) Enlarged view of mesh in the pipe bend

Fig. 1. Meshing of pipe bend geometry.

\subsection{Boundary Conditions and Solution Strategy}

Simulations are performed with the assumption that the flow is steady. The calculation domain is bounded by three faces, the inlet boundary, the wall boundary, and the outlet boundary. EulerianEulerian model is considered for the simulation.
Water is considered as a primary phase and zinc tailings particles are considered as the secondary phases. For modeling the flow with multi-sized particulate slurry, six secondary phases of different mean particle diameter and initial solid volume fraction are considered. The initial volume fraction of the solids of different sizes is selected based on the PSD of the multisized zinc tailings slurry used by Mishra et al. (1998a) as shown in Table 1. Velocity inlet and outflow are set as a boundary condition at the inlet and outlet of the flow domain, respectively. The initial velocity of all the phases at the inlet is set to be equal. The initial volume fraction of solids for each solid phase is set based on PSD. Table 2 shows the solution strategy adopted for the numerical simulations.

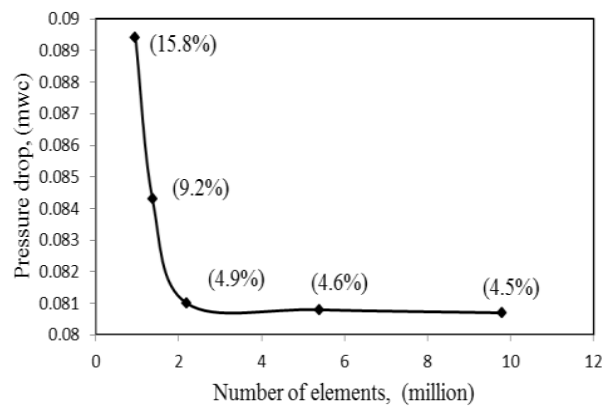

Fig. 2. Pressure drop variation with the number of mesh elements.

Table 1 Particle size distribution of multisized zinc tailing particles Mishra et al. (1998a)

\begin{tabular}{|c|c|c|c|c|c|c|c|}
\hline $\begin{array}{c}\text { Solid } \\
\text { Size, } \\
(\mu \mathrm{m})\end{array}$ & $>850$ & 300 & 210 & 150 & 106 & 75 & 30 \\
\hline$\%$ Finer & 100 & 96.3 & 85.1 & 78.6 & 63.7 & 51.4 & 2 \\
\hline $\begin{array}{c}\text { Mean } \\
\text { Size } \\
(\mu \mathrm{m})\end{array}$ & 575 & 255 & 180 & 128 & 90.5 & 37.5 \\
\hline $\begin{array}{c}\text { Wt. } \\
\text { Fract. } \\
(\%)\end{array}$ & 3.7 & 10.4 & 7.3 & 14.9 & 12.3 & 49.4 \\
\hline
\end{tabular}

\subsection{Model Validation}

The numerically obtained pressure drop in meters of water column (mwc) between 2D upstream and 2D downstream of the bend is compared with the available experimental results (Mishra et al. 1998) as shown in Fig. 3. The comparison of the results is plotted for all the four weighted solid concentrations of $9.82 \%, 20.32 \%, 30.21 \%$ and $44.26 \%$ and five flow velocities of $2.25 \mathrm{~m} / \mathrm{s}, 2.55$ $\mathrm{m} / \mathrm{s}, 2.86 \mathrm{~m} / \mathrm{s}, 3.2 \mathrm{~m} / \mathrm{s}$ and $3.5 \mathrm{~m} / \mathrm{s}$. It is observed that the pressure drop increases with increase in velocity as well as solid concentration. At low velocities, the increase in pressure drop with the increase in solid concentration is less compared to higher flow velocity. The predicted pressure drop results show good agreement with the experimental results (Mishra et al. 1998a). The maximum 
deviation in the predicted and experimental results is within $\pm 6 \%$.

Table 2 Boundary conditions and solution parameters used for the numerical simulation

\begin{tabular}{|c|c|}
\hline Parameters & Modeling \\
\hline Components & $\begin{array}{l}\text { Pipe inlet to bend, bend, Pipe } \\
\text { exit to bend }\end{array}$ \\
\hline Grid type & $\begin{array}{c}\text { Structured: Multi-block } \\
\text { Hexahedral }\end{array}$ \\
\hline Analysis type & Steady \\
\hline $\begin{array}{c}\text { Multiphase } \\
\text { model }\end{array}$ & Eulerian-Eulerian \\
\hline No. of Phases & $\begin{array}{c}\text { Primary: One (water) } \\
\text { Secondary: Six (zinc tailings) }\end{array}$ \\
\hline $\begin{array}{l}\text { Boundary } \\
\text { conditions }\end{array}$ & $\begin{array}{l}\text { Inlet: Velocity, Volume fraction } \\
\text { Outlet: Outflow }\end{array}$ \\
\hline $\begin{array}{l}\text { Discretization } \\
\text { scheme }\end{array}$ & $\begin{array}{c}\text { Momentum Eq.: First order } \\
\text { upwind (FOU) } \\
\text { Volume fraction: FOU } \\
\text { Turbulent kinetic energy: FOU } \\
\text { Turbulent dissipation energy: } \\
\text { FOU }\end{array}$ \\
\hline $\begin{array}{c}\text { Under } \\
\text { relaxation factor }\end{array}$ & $\begin{array}{c}\text { Pressure: } 0.3 \\
\text { Momentum equation: } 0.7 \\
\text { Volume fraction: } 0.5 \\
\text { Turbulent kinetic energy: } 0.8 \\
\text { Turbulent dissipation energy: } 0.8\end{array}$ \\
\hline $\begin{array}{l}\text { Pressure } \\
\text { velocity } \\
\text { coupling } \\
\text { scheme }\end{array}$ & Phase Coupled SIMPLE \\
\hline $\begin{array}{l}\text { Turbulence } \\
\text { models }\end{array}$ & $\begin{array}{c}\text { Standard } \mathrm{k}-\varepsilon \text { with dispersed } \\
\text { phase. }\end{array}$ \\
\hline $\begin{array}{l}\text { Convergence } \\
\text { control }\end{array}$ & $\begin{array}{l}\text { rms of mass, momentum, } \\
\text { volume fraction and turbulent } \\
\text { parameters } \leq 1.0 \mathrm{E}-5\end{array}$ \\
\hline
\end{tabular}

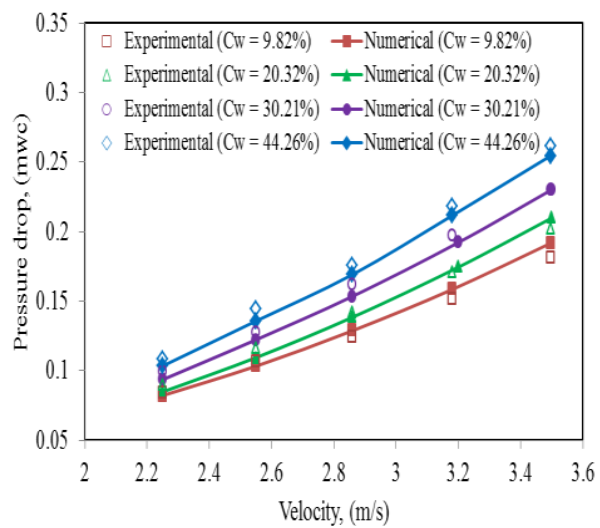

Fig. 3. Comparison of predicted pressure drop to the experimental results of Mishra et al.

(1998a) for different weighted solid concentration and velocity.

\section{RESUlts AND DisCUSSION}

\subsection{Pressure Distribution}

The effect of flow velocity and solid concentration

on the absolute pressure inside the bend are determined by plotting the contours of absolute pressure variation in the bend geometry at the mid horizontal plane as shown in Fig. 4 and Fig. 5. It is observed that upstream of the bend inlet, the reduction in absolute pressure is uniform. As the flow enters into the bend geometry, a pressure gradient is established between the inner and outer wall of the bend. This may be attributed to the outward centrifugal force experienced by the flow during the flow passage of the bend that pushes the flow towards the outer wall of the bend and creates a pressure variation along the inner and outer wall of the bend. It is also observed that the difference in pressure variation at inner and outer wall increases as the flow progresses towards the bend exit. This may be attributed to the presence of secondary flows inside the bend. Similar effect of pressure variations at the inner and outer wall of the bend were also observed earlier (Kaushal et al. 2013; Singh et al. 2018).

Figure 4 shows the contours of absolute pressure variation inside the bend for the velocity range of $2.25 \mathrm{~m} / \mathrm{s}$ to $3.5 \mathrm{~m} / \mathrm{s}$ at the weighted solid concentration of $44.26 \%$. It is observed that the increase in flow velocity increases the difference in absolute pressure at the inner and outer wall of the bend. It may be due to the increase in frictional losses with increase in velocity (Nayak et al. 2017). Figure 5 shows contours of absolute pressure variation inside the bend for the weighted solid concentration of $9.82 \%$ to $44.26 \%$ at the inflow velocity of $3.5 \mathrm{~m} / \mathrm{s}$. It is observed that the increase in solid concentration increases the reduction in absolute pressure during the flow inside the bend. It may be due to the increase in the particle-particle interaction with the increase in solid concentration (Verma et al. 2006).

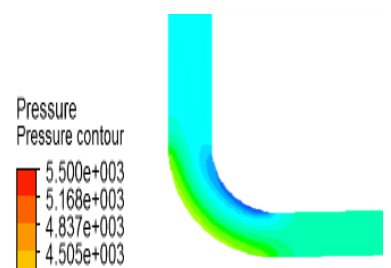

(a) $2.25 \mathrm{~m} / \mathrm{s}$

$-4.174 e+0$

$-3.842 \mathrm{e}+003$

$-3.511 e+003$
$-3.179 \mathrm{e}+003$

$-3.179 e+003$
$-2.847 e+003$

$2.516 \mathrm{e}+003$

$21840+003$

$2.184 \mathrm{e}+003$

$1.853 \mathrm{e}+003$

$1.521 \mathrm{e}+003$

$1.189 \mathrm{e}+003$

$8.579 \mathrm{e}+002$

$5.263 \mathrm{e}+002$

$1.947 \mathrm{e}+002$

$-1.368 \mathrm{e}+002$

$4.684 \mathrm{e}+002$

$-8000 \mathrm{e}+002$

[Pa]

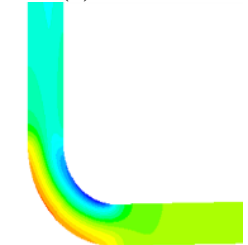

(c) $2.86 \mathrm{~m} / \mathrm{s}$

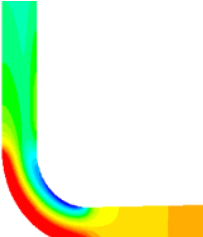

(d) $3.2 \mathrm{~m} / \mathrm{s}$

(e) $3.5 \mathrm{~m} / \mathrm{s}$

(b) $2.55 \mathrm{~m} / \mathrm{s}$

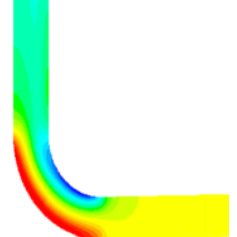

Fig. 4. Absolute pressure variation with flow velocity in pipe bend at the mid horizontal plane at $\mathrm{C}_{\mathrm{w}}=\mathbf{4 4 . 2 6 \%}$. 
R. Tarodiya et al. / JAFM, Vol. 13, No. 4, pp. 1311-1321, 2020.

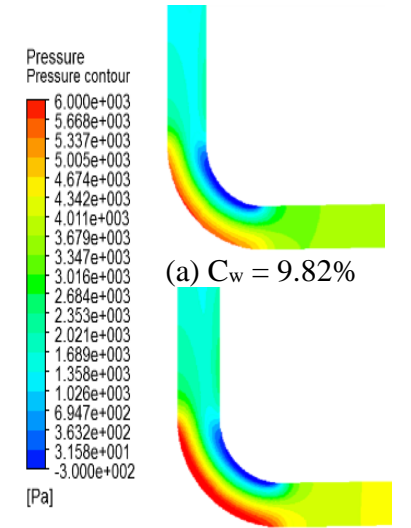

(c) $\mathrm{C}_{\mathrm{w}}=30.21 \%$

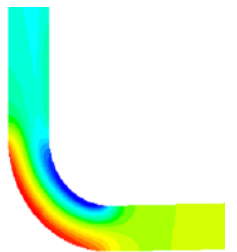

(b) $\mathrm{C}_{\mathrm{w}}=20.32 \%$

(d) $\mathrm{C}_{\mathrm{w}}=40.26 \%$

Fig. 5. Absolute pressure variation with the solid concentration in pipe bend at the mid horizontal plane at $\mathrm{V}=3.5 \mathrm{~m} / \mathrm{s}$.

Particle

size

(a) Bend Inlet

(b) Bend

Center

(c) Bend Exit

(d) $5 \mathrm{D}$

(e) $10 \mathrm{D}$

$(\mu \mathrm{m})$

(i) 575
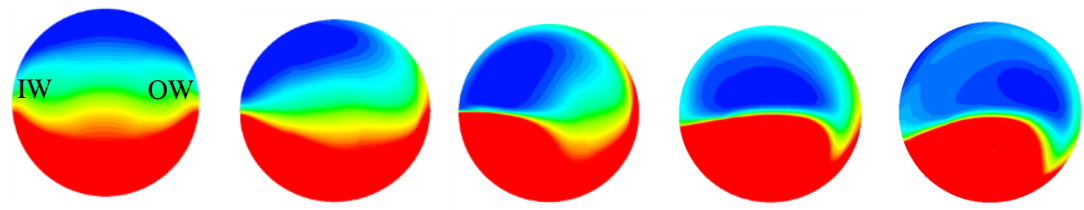

$\mathrm{C}_{\mathrm{r} /} / \mathrm{C}_{\mathrm{r}}$
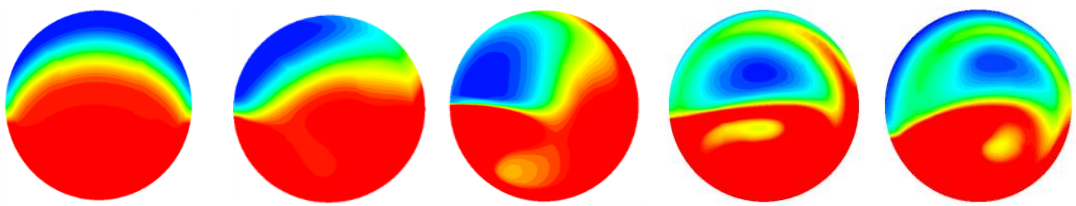

(iii) 180
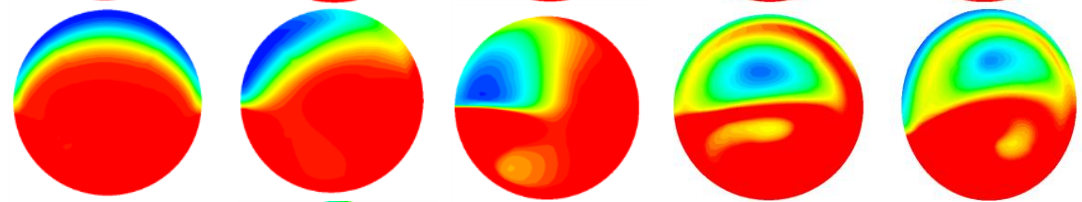

(iv) 128
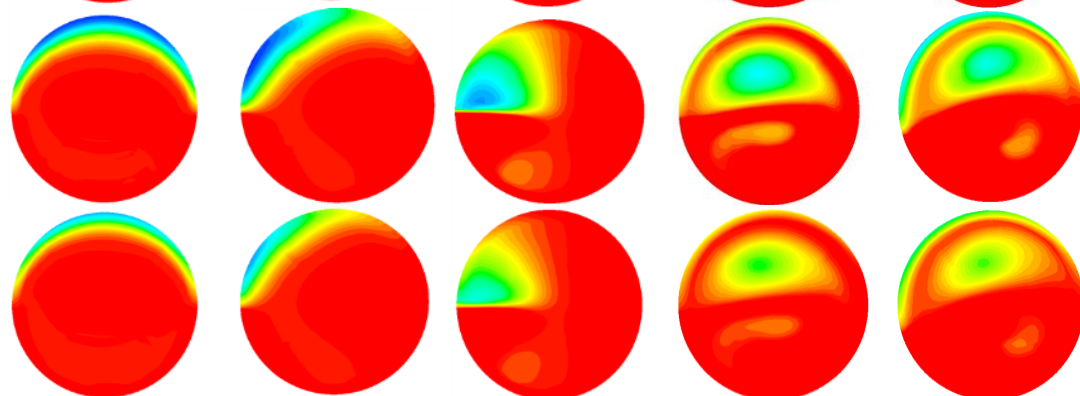

(v) 90.5
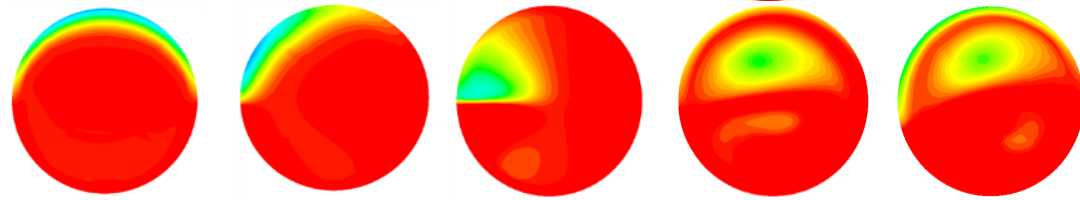

(vi) 37.5
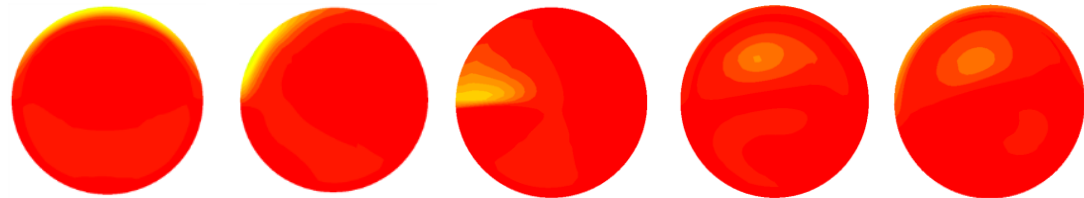

Fig. 6 Cross-sectional normalized concentration contours (outer wall at right side) of each size particles at different angular positions, 5D, 10D distances from bend exit at velocity $=2.25 \mathrm{~m} / \mathrm{s}$, $\mathrm{Cw}=\mathbf{9 . 8 2 \%}$

\subsection{Particle Distribution}

Figures (6-8 show the contours of the crosssectional normalized concentration which is the ratio of particle volume fraction at the location $\left(\mathrm{C}_{\mathrm{vi}}\right)$ to the particle initial volume fraction $\left(\mathrm{C}_{\mathrm{v}}\right)$ for each solid phase. These are plotted at the bend inlet $\left(0^{\circ}\right)$, bend center $\left(45^{\circ}\right)$, bend exit $\left(90^{\circ}\right)$, at five times pipe 


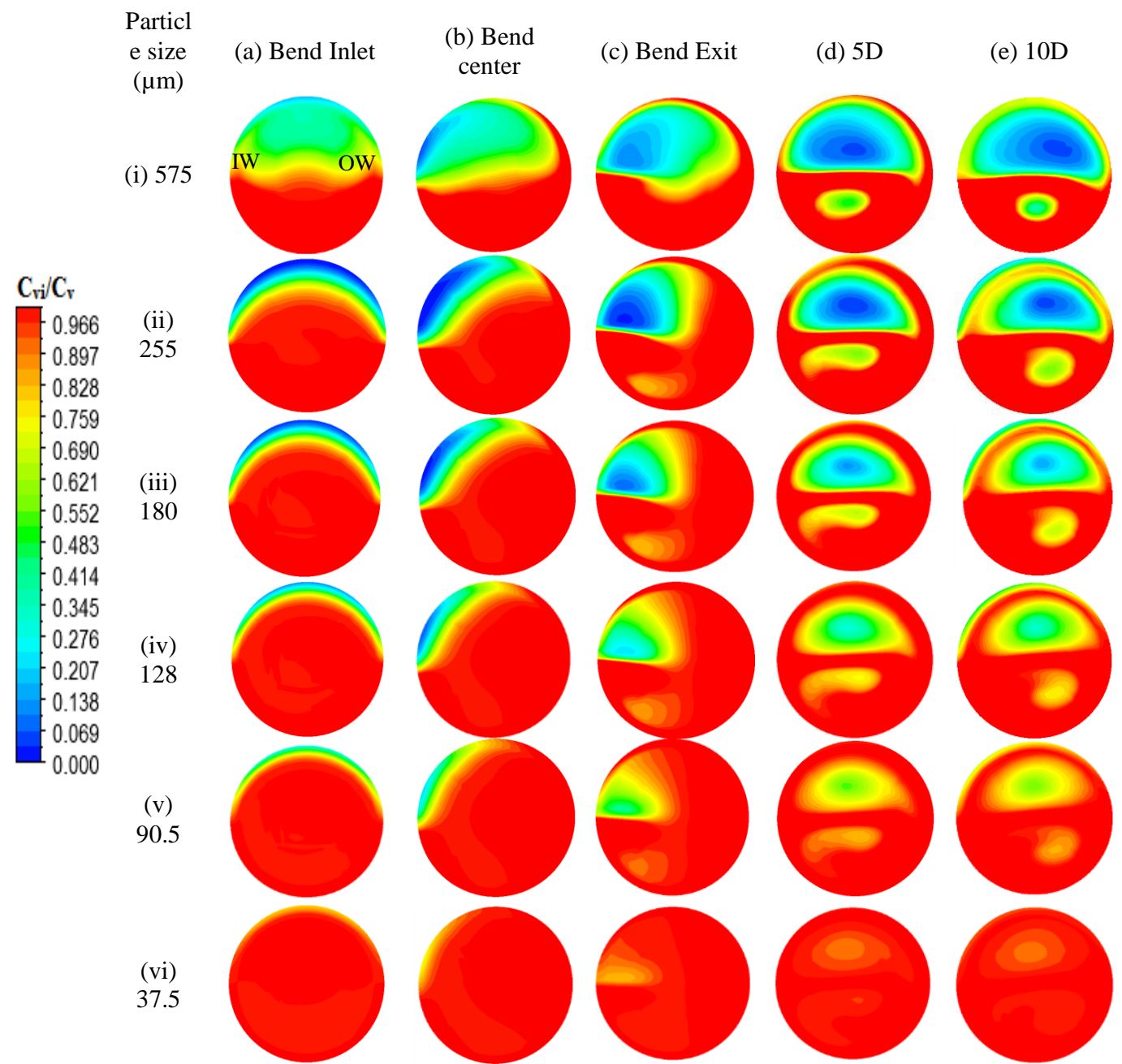

Fig. 7 Cross-sectional normalized concentration contours (outer wall at right side) of each size particles at different angular positions, 5D, 10D distances from bend exit at velocity $=3.5 \mathrm{~m} / \mathrm{s}, \mathrm{Cw}=$ $9.82 \%$.

diameter (5D) and ten times pipe diameter (10D) distance downstream to bend exit. In these figures, the left-hand side represents the inner wall whereas the right hand side represents the outer wall of the bend. Figures 6 and 7 show the suspension characteristics of multi-sized particulate slurry inside the bend at the velocity of $2.25 \mathrm{~m} / \mathrm{s}$ and 3.5 $\mathrm{m} / \mathrm{s}$, respectively at $9.82 \%$ weight concentration. Figure 8 shows the suspension characteristics of multi-sized particulate slurry at the velocity of 2.25 $\mathrm{m} / \mathrm{s}$ and $44.26 \%$ weight concentration.

It is observed from Fig. 6 that the suspension characteristics of the particles at different locations inside the bend are dissimilar. At the bend inlet (Fig. 6a), the concentration distribution of all the particles is skewed except the fine size particles of size $37.5 \mu \mathrm{m}$. It may be due to settling behavior of particles in the presence of gravity and therefore high concentration is observed at the bottom of the bend inlet. As the flow passes through the bend, the centrifugal forces and the secondary flows influence the distribution of the particles (Kaushal et al. 2012; Nayak et al. 2017). At the bend center (Fig. 6b), the particles moved towards the upper side at the outer wall of the bend. This may be attributed to the centrifugal force along with the effect of bend curvature. Then, the presence of secondary flow moves the particle from the outer to the inner wall. These effects increase up to the bend exit (Fig. 6c) and the particle concentration is higher at the upper end of the bend outer wall and the bottom half. At 5D position downstream to the bend exit (Fig. 6d) the secondary flow may still affect the suspension characteristics of the particles. The low concentration zone of particles is shifted to the central core, and the particle concentration near the wall gets improved. The effect of secondary flow is reduced as seen at the $10 \mathrm{D}$ position from the bend exit (Fig. 6e). Further, on comparing the suspension behavior of multi-sized particles, it is observed that the fine size particles of size $37.5 \mu \mathrm{m}$ are homogeneously suspended, whereas non-uniformity in particle distribution is clearly observed for higher size particles. For particle size up to $255 \mu \mathrm{m}$, the higher concentration of particles is seen at the outer wall and the bottom of the bend. This may be attributed to the effect of centrifugal force and the 
R. Tarodiya et al. / JAFM, Vol. 13, No. 4, pp. 1311-1321, 2020.

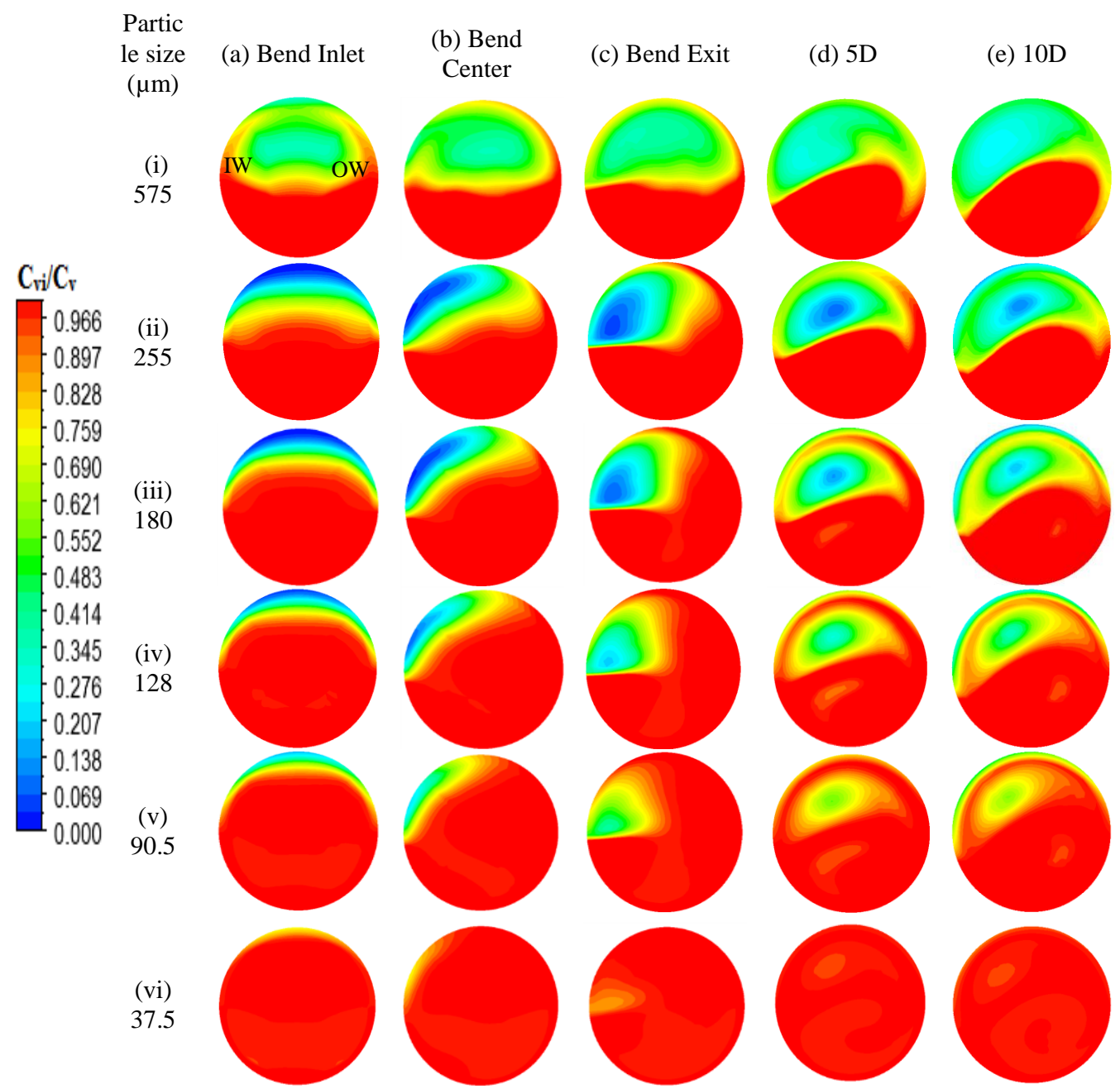

Fig. 8 Cross-sectional normalized concentration contours (outer wall at right side) of each size particles at different angular positions, 5D, 10D distances from bend exit at velocity $=2.25 \mathrm{~m} / \mathrm{s}$, $\mathrm{Cw}=44.26 \%$.

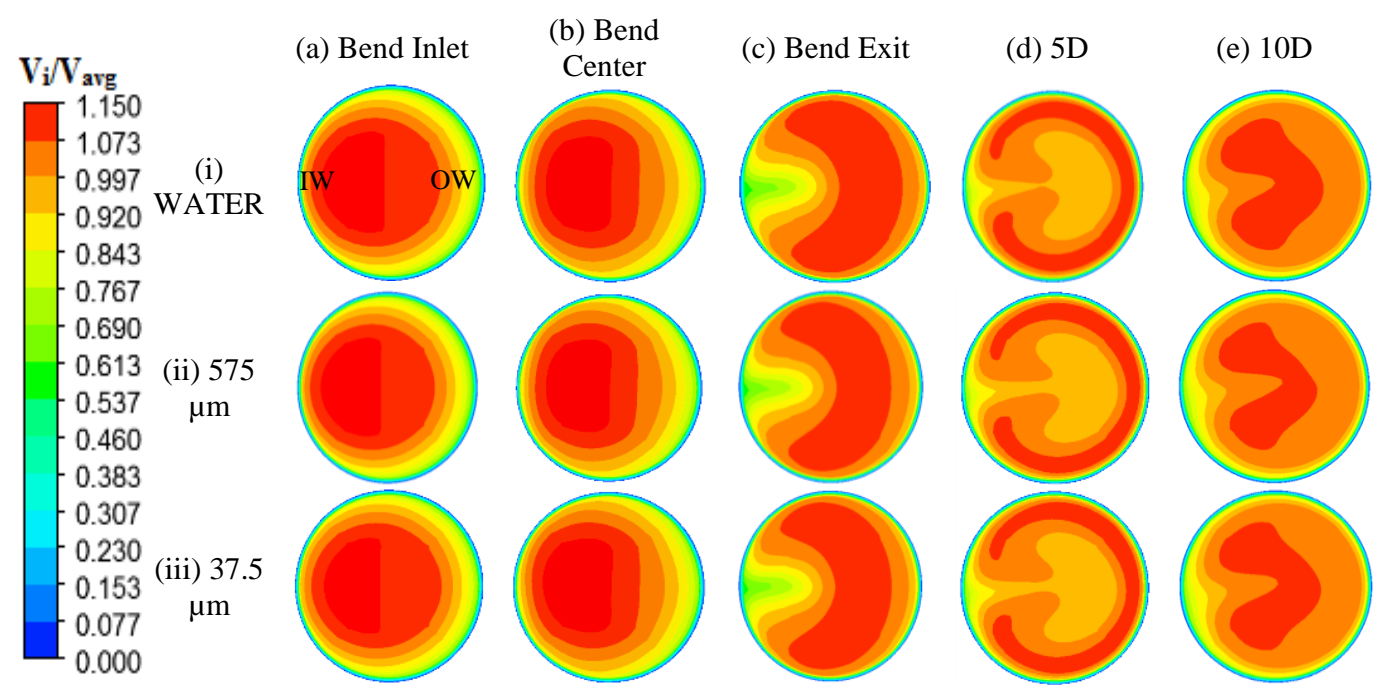

Fig. 9 Cross-sectional normalized velocity contours (outer wall at right side) for water, particle size of $575 \mu \mathrm{m}$ and $37.5 \mu \mathrm{m}$ at different angular positions, 5D, 10D distances from bend exit at velocity $=3.5 \mathrm{~m} / \mathrm{s}, \mathrm{Cw}=9.82 \%$. 
secondary flows. The distribution of $575 \mu \mathrm{m}$ size particles shows no significant change and remains at the bottom throughout the flow passage. This may be attributed to the dominance of gravity effect for this size of particles at the flow velocity of 2.25 m/s. Further, comparison of Fig. 6 and Fig. 7 shows that, with the increase in flow velocity, the particle distribution at the bend inlet improves significantly. The concentration of particles increases more towards the upper half of the outer wall of the bend. The suspension of the particles of size $575 \mu \mathrm{m}$ is also improved as some particles are flowing near the upper part of the outer wall. This may be attributed to the increase in the kinetic energy of the particles as more number of particles are able to overcome the gravitational effects and remain suspended in the carrier fluid.

To observe the effect of increase in efflux concentration on the particle distribution, the contours of normalized solid concentration at different cross-sections inside the bend are compared using Fig. 6 and Fig. 8. It is observed that the increase in solid concentration improves particle distribution. This may be attributed to the increase in the interference effects between the particles with increase in solid concentration (Chen et al. 2009). Mishra et al. (1998b) also reported the improvement in the solid distribution with the increase in efflux concentration at constant velocity. Further, with increase in concentration, it is observed that even the distribution of bigger size particles like $255 \mu \mathrm{m}$ and $575 \mu \mathrm{m}$ also improved. This may be attributed to the increase in the number of fine size particles $(37.5 \mu \mathrm{m})$ in the multisized slurry with the increase in total solid concentration. The improvement in particle distribution of bigger size particles with the addition of finer size in the straight pipeline was also reported by Kumar et al. (2008).

\subsection{Velocity Distribution}

Figure 9 shows the contours of normalized velocity which is the ratio of the phase velocity at the location $\left(\mathrm{V}_{\mathrm{i}}\right)$ to the bulk mean flow velocity $\left(\mathrm{V}_{\mathrm{avg}}\right)$ for water and the particles of size $575 \mu \mathrm{m}$ and 37.5 $\mu \mathrm{m}$ at the velocity of $3.5 \mathrm{~m} / \mathrm{s}$ and $9.82 \%$ weight concentration. It is observed that at the bend inlet (Fig. 9a) the maximum velocity is at the central core which agrees with the general understanding of the flow through the circular pipes. At the bend center (Fig. 9b) the effect of secondary flows is not significant, and a small deviation in the velocity contours is observed in comparison to bend inlet. At bend exit (Fig. 9c), the outer wall of the bend possesses a higher magnitude of velocity which shows that the secondary flow is dominant in the region between the bend center and the bend exit. It affects majorly up to 5D (Fig. 9d) distance from the bend exit, where the maximum velocity is observed near the outer side of the pipe wall. As the distance increases further, the intensity of secondary flows reduces and at 10D (Fig. 9e) distance from the bend exit the velocity of the phases is again maximum at the central core of the pipe. It is also observed that for different phases the velocity distribution is of the similar nature and variation with respect to the different locations is also similar. It indicates that the effect of slip between the particle and the carrier fluid is negligible in the present numerical simulations.

\section{CONClusion}

Numerical modeling of the multi-sized particulate slurry flow in a conventional $90^{\circ}$ bend of radius ratio 4 is performed. Granular Eulerian-Eulerian model with six secondary phases of different size particles along with the primary phase as water are considered for the numerical simulations. On the basis of the present study the following conclusions can be drawn:

- The numerical modeling of the pipe bend with multisized particulate slurry predicted the head loss close to experiments. The maximum deviation is within $\pm 6 \%$ for the present range of investigation. The effect of increase in flow velocity and solid concentration on pressure drop are reasonably simulated with the numerical model.

- The simulation with multisized particles captured the improvement in non-uniformity in suspension due to more number of finer size particles at higher concentrations concurrent with the experimental observations.

- Within the multisized particulate slurry as the particle size increases the effect of secondary flows becomes more and more dependent on PSD.

- Increase in the velocity enhances the homogeneous suspension of all size particles in the multisized slurry. However, a higher velocity of flow is needed for the complete suspension of bigger size particles in the slurry. Hence the critical velocity should be estimated by considering the settling of coarse particles in a multisized slurry.

\section{ACKNOWLEDGEMENTS}

The authors are thankful to the Ministry of Human Resource and Development (MHRD), Government of India for financial support.

\section{REFERENCES}

Chen, L., Y. Duan, W. Pu and C. Zhao (2009). CFD simulation of coal-water slurry flowing in horizontal pipelines. Korean Journal of Chemical Engineering 26(4), 1144-1154.

Dalla Valle, J. M. (1948). Micromeritics. Pitman, London.

Ding, J. and D. Gidaspow (1990). A bubbling fluidization model using kinetic theory of granular flow. AIChE Journal 36(4), 523-538.

Fluent (2006). User's Guide Fluent 6.2. Fluent Incorporation, USA. 
Garside, J. and M. R. Al-Dibouni (1977). VelocityVoidage relationships for fluidization and sedimentation. Industrial \& Engineering Chemistry Process Design and Development 16, 206-214.

Gidaspow, D., R. Bezburuah, and J. Ding (1992). Hydrodynamics of circulating fluidized beds, kinetic theory approach. In Fluidization VII, Proceedings of the $7^{\text {th }}$ Engineering Foundation Conference on Fluidization, Brisbane, Australia, 75-82.

Gopaliya, M. K. and D. R. Kaushal (2015). Analysis of effect of grain size on various parameters of slurry flow through pipeline using CFD. Particulate Science and Technology 33(4), 369-384.

Kaushal, D. R. and Y. Tomita (2002). Solids concentration profiles and pressure drop in pipeline flow of multisized particulate slurries. International Journal of Multiphase Flow 28, 1697-1717.

Kaushal, D. R., A. Kumar, Y. Tomita, S. Kuchii, and H. Tsukamoto (2013). Flow of monodispersed particles through horizontal bend. International Journal of Multiphase Flow 52, 71-91.

Kaushal, D. R., T. Thinglas, Y. Tomita, S. Kuchii, and H. Tsukamoto (2012). CFD modeling for pipeline flow of fine particles at high concentration. International Journal of Multiphase Flow 43, 85-100.

Kumar, U., S. N. Singh and V. Seshadri (2008). Prediction of flow characteristics of bimodal slurry in horizontal pipe flow. Particulate Science and Technology 26, 361-379.

Lun, C. K. K., S. B. Savage, D. J. Jefferey, and N. Chepurniy (1984). Kinetic theories for granular flow: inelastic particles in couette flow and slightly inelastic particles in a general flow field. Journal of Fluid Mechanics 140, 223256.

Messa, G. V. and S. Malavasi (2014). Numerical prediction of particle distribution of solidliquid slurries in straight pipes and bends. Engineering Applications of Computational Fluid Mechanics 8(3), 356-372.

Mishra, R., S. N. Singh and V. Seshadri (1998a). Pressure drop across conventional and diverging-converging pipe bends in the flow of multisized particulate slurries. Indian Journal of Engineering and Material Sciences 5, 9-14.

Mishra, R., S. N. Singh and V. Seshadri (1998b). Study of wear characteristics and solid distribution in constant area and erosion- resistant long-radius pipe bends for the flow of multisized particulate slurries. Wear 217, 297306.

Mukhtar, A., S. N. Singh, and V. Seshadri (1995). Pressure drop in a long radius $90^{\circ}$ horizontal bend for the flow of multisized heterogeneous slurries. International Journal of Multiphase Flow 21(2), 329-334.

Nayak, B. B., D. Chatterjee, and A. N. Mullick (2017). Numerical prediction of flow and heat transfer characteristics of water-fly ash slurry in a $180^{\circ}$ return pipe bend. International Journal of Thermal Science 113, 100-115.

Schaeffer, D. G. (1987). Instability in the evolution equations describing incompressible granular flow. Journal of Differential Equations 66, 1950 .

Singh, J. P., S. Kumar, and S. K. Mohapatra (2018). Simulation and optimization of coal-water slurry suspension flow through $90^{\circ}$ pipe bend using CFD. International Journal of Coal Preparation and Utilization.

Singh, J. P., S. Kumar, and S. K. Mohapatra (2019). An experimental study on head loss characteristics of pipe bends for flow of coalwater slurry at high solid concentration. IMechE Part E Journal of Process Mechanical Engineering 0(0), 1-11.

Syamlal, M. (1987). The particle-particle drag term in a multi-particle model of fluidization. National Technical Information Service, Springfield, VA, DOE/MC/21353-2373, NTIS/DE87006500.

Syamlal, M., W. Rogers, and T. J. O'Brien (1993). MFIX Documentation: Volume 1, Theory Guide. National Technical Information Service, Springfield, VA, DOE/METC9411004, NTIS/DE9400087.

Tarodiya, R. and B. K. Gandhi (2017). Hydraulic performance and erosive wear of centrifugal slurry pumps - a review. Powder Technology $305,27-38$.

Tarodiya, R. and B. K. Gandhi (2019). Numerical simulation of a centrifugal slurry pump handling solid-liquid mixture: effect of solids on flow field and performance. Advanced Powder Technology 30, 2225-2239.

Verma, A. K., S. N. Singh, and V. Seshadri (2006). Pressure drop for the flow of high concentrated solid-liquid mixture across $90^{\circ}$ horizontal conventional circular pipe bend. Indian Journal of Engineering and Material Sciences 13, 477-483. 